\title{
Seismic scattering and absorption mapping of debris flows, feeding paths, and tectonic units at Mount St. Helens volcano
}

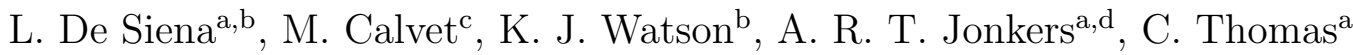 \\ ${ }^{a}$ University of Münster, Institut für Geophysik, Correnstrasse 24, 48149 Münster, \\ Germany (lucadesiena@abdn.ac.uk). \\ ${ }^{b}$ University of Aberdeen, School of Geosciences, Geology and Petroleum Geology, Meston \\ Building, King's College, Aberdeen AB24 3UE Scotland, UK. \\ ${ }^{c}$ Institut de Recherche en Astrophysique et Planétologie, Université Paul Sabatier / \\ CNRS, 14 Avenue Edouard Belin, 31400 Toulouse, France. \\ ${ }^{d}$ Department of Mathematics, University of Liverpool / Liverpool, UK.
}

\begin{abstract}
Frequency-dependent peak-delay times and coda quality factors have been used jointly to separate seismic absorption from scattering quantitatively in Earth media at regional and continental scale; to this end, we measure and map these two quantities at Mount St. Helens volcano. The results show that we can locate and characterize volcanic and geological structures using their unique contribution to seismic attenuation. At $3 \mathrm{~Hz}$ a single high-scattering and high-absorption anomaly outlines the debris flows that followed the 1980 explosive eruption, as deduced by comparison with remote sensing imagery. The flows overlay a NNW-SSE interface, separating rocks of significant varying properties down to $2-4 \mathrm{~km}$, and coinciding with the St. Helens Seismic Zone. High-scattering and high-absorption anomalies corresponding to known locations of magma emplacement follow this signature under the volcano, showing the important interconnections between its feed-
\end{abstract}


ing systems and the regional tectonic boundaries. With frequency increasing from 6 to $18 \mathrm{~Hz}$ the NNW-SSE tectonic/feeding trends rotate around an axis centered on the volcano in the direction of the regional-scale magmatic arc (SW-NE). While the aseismic high-scattering region WSW of the volcano shows no evidence of high absorption, the regions of highest-scattering and absorption are consistently located at all frequencies under either the eastern or the south-eastern flank of the volcanic edifice. From the comparison with the available geological and geophysical information we infer that these anomalies mark both the location and the trend of the main feeding systems at depths greater than $4 \mathrm{~km}$.

Keywords: Seismic scattering, Seismic absorption, Volcano imaging, Tectonic structures, Debris flows, Feeding systems

\section{1}

\section{Introduction}

2 Seismic attenuation measurements provide complementary information to 3 velocity tomography on the state of volcanic media, thereby increasing our in4 sight into their complex structure and composition. Knowing the mechanism 5 with which seismic waves lose their energy in space, time, and frequency in 6 the volcanic crust (either scattering or absorption) is crucial to improve seis7 mic images of feeding paths and tectonic structures. The lateral variations 8 in seismic attenuation induced by these two mechanisms can be quantified 9 by (1) the peak-delay time of shear waves, defined as the lapse-time cor-

\footnotetext{
${ }^{1}$ Corresponding author: Luca De Siena (lucadesiena@abdn.ac.uk)
} 
responding to the maximum of the seismogram envelope and (2) the coda quality factor $Q_{c}$, which quantifies the decay rate of the coda envelope with increasing lapse-time $[1,2,3,4]$.

Researchers were able to illuminate tectonic structures by mapping these two quantities, as done e.g. by Calvet et al. [3] in the Pyrenean mountain range. Takahashi et al. [1] have shown that peak-delay times increase as a consequence of strong forward scattering when seismic waves cross quaternary volcanoes embedded in the Japanese crust. Also in Japan, Carcolé and Sato [5] have obtained high- resolution maps of seismic scattering and absorption by using the Multiple Lapse Time Window Analysis method [6, 7]. Their results demonstrate that the spatial variations of intrinsic absorption and $Q_{c}$ at sufficient lapse-times from the origin time of the earthquake are highly correlated. At both regional and continental scale systematically higher peak-delay times and lower $Q_{c}$ measurements therefore mark the most highly heterogeneous and absorbing structures.

We measure peak-delay times and $Q_{c}$ to map frequency-dependent lateral variations of $S$ wave scattering and absorption in the highly-heterogeneous crust under Mount St. Helens volcano (hereafter MSH). We follow an approach similar to that of Calvet et al. [3] to separate seismic scattering from seismic absorption. The only modification is that we measure $S$ wave peakdelay times with respect to the $P$ wave arrival. Our main assumptions are that (1) absorption strongly affects late lapse-time $Q_{c}$ measurements and (2) peak-delay times and $Q_{c}$ sensitivities are distributed uniformly along the 3D seismic ray [3]. The results of statistical analyses and the knowledge provided by geophysics, geology, and remote sensing in this well known area (see, e.g., 
De Siena et al. [8] for a complete review) will shed light on the effects that different Earth media produce on coda intensities. The outcomes will both improve our understanding of how heterogeneous structures influence coda waves and give us a novel way to depict volcanic media at different scales, from shallow debris flows and geological units to deep feeding and tectonic systems.

\section{Materials and methods}

\subsection{Data and $P$ wave seismic heterogeneity}

We use 451 high-quality vertical velocity waveforms produced by 64 earthquakes with magnitudes between 1.5 and 2.7 located around MSH (Fig. 1a-c). The waveforms are recorded at 13 stations of the Pacific Northwest Seismic Network between 2000 and 2003, before the explosive eruption of the volcano in 2004 (Fig. 1a). Hypocentral distances of lengths spanning between 5 and $60 \mathrm{~km}$ are measured along the seismic rays, traced using a Thurbermodified ray-bending approach in the velocity model of Waite and Moran [9]. After the deconvolution from the instrument response the seismograms are filtered in 4 frequency bands $(2-4 \mathrm{~Hz}, 4-8 \mathrm{~Hz}, 8-16 \mathrm{~Hz}, 16-32$ $\mathrm{Hz})$. We compute the root mean square $(r m s)$ of the velocity waveforms and smooth the time series with a moving time-window whose duration is twice the central period of each frequency band. Finally, we calculate the seismic envelopes/intensities as the sum of the rms and of its Hilbert transform.

Using the velocity model of Waite and Moran [9] we also obtain a 2D map of the rms of the $P$ wave velocity fluctuations (Fig. 2a, $\varepsilon$ ), a direct measurement of $P$ wave heterogeneity, following the approach described by 
De Siena et al. [10]. An exponential autocorrelation functions (ACF) is calculated using the $P$ wave velocity tomograms [9] as measurements of the velocity wave field from the surface to depths of $10 \mathrm{~km}$, in the regions of maximum resolution. To remove the depth dependence we fit the 21 vertical high-resolution (0.5 km depth slices) velocity measurements obtained at each point of the 2D map (Fig. 1a) by a Nth order average polynomial $V(z)=$ $a_{0}^{*}+a_{1}^{*} z+a_{2}^{*} z^{2}+\ldots+a_{N}^{*} z^{N}$. Assuming identical variances for the depthdependent measurements we obtain the degree $\mathrm{N}=4$ of the polynomial and its coefficients $a^{*}$ by using the Schwartz information criterion. The mean squared velocity fluctuations $\left(\varepsilon^{2}\right)$ are estimated by measuring the maximum of the ACF obtained after detrending for this polynomial [10]. We will only discuss the points of the velocity model where we obtain random velocity fluctuations with a Lilliefors (Kolmogorov-Smirnov) normality test at a 5\% significance level [10], which correspond to the $76 \%$ of our starting measurements.

A generalized extreme value distribution of second type with positive shape parameter (i.e., a Fréchét distribution) fits the $\varepsilon$ data (Fig. 2b). The Fréchét distribution is the limit distribution of maxima of a sequence of nongaussian independent and identically distributed random variables, like the velocity fluctuations. It provides a location parameter (or, more simply, a spatially averaged $r m s$ ) of 0.0088 , with a scale parameter (standard deviation) of 0.025 . The small positive shape parameter $(0.18 \pm 0.1)$ shows that only few observations largely differ from the location parameter. A correlation function is also defined by a correlation length, which may be measured as the length at which the ACF reaches half of its maximum [e.g. 10]. At Campi Flegrei, De Siena et al. [11] show that typical variations of the corre- 
lation length produce small variations in broadening of the coda wave field. Since this quantity is also affected by larger uncertainties we consider $\varepsilon$ as the best parameter to map $P$ wave heterogeneity at MSH (Fig. 2a). The map shows a north-trending high- $\varepsilon$ anomaly starting under the northeastern part of the volcanic edifice and a second high- $\varepsilon$ anomaly located south-south-west of the MSH volcanic edifice.

\subsection{Peak-delay time mapping}

Various definitions of peak-delay time $\left(t_{r}\right)$ have been used in literature to quantify the strength of scattering due to random heterogeneities along the seismic ray-path. Its most general definition is the time-lag between the $S$ wave onset and the maximum of the $S$ wave amplitude $[1,12,3]$. As $S$ waves are difficult (if not impossible) to pick on MSH recordings, we define $t_{r}$ as the time between the $P$ wave arrival $\left(t_{p}\right)$ and the maximum of the envelope (Fig. 3).

In Fig. 4a, we plot $\log _{10}\left(t_{r}\right)$ as a function of $\log _{10}\left(t_{p}\right)$ (gray circles) for a selected number of peak-delay times measured on the waveforms in our dataset. Using a Markov approximation of the parabolic equation, Saito et al. [13] have demonstrated that in heterogeneous media the logarithm of $t_{r}$ linearly increases with the logarithm of $t_{p}$ at all frequencies. However, our measurements are taken in a highly-heterogeneous volcanic region; this linear dependence therefore imperfectly matches the true physical peak-delay model due to regional variations of (1) $P$ and $S$ wave relative velocities and (2) the strength of the $S$ wave heterogeneities, which produce high and localized $S$ wave scattering $[14,11]$.

But relative lateral variations of seismic velocities cannot explain peak- 
delay time fluctuations of the order of those measured at different $t_{p}$. The theoretical difference between the $S$ wave travel time $\left(t_{s}\right)$ and $t_{p}$ in an homogeneous medium is given by:

$$
\log _{10}\left[t_{s}-t_{p}\right]=\log _{10}\left[\left(\frac{V_{p}}{V_{s}}-1\right) t_{p}\right]=\log _{10}\left[\left(\frac{V_{p}}{V_{s}}-1\right)\right]+\log _{10}\left[t_{p}\right]=C+\log _{10}\left[t_{p}\right] .
$$

Feasible variations of the velocity ratio $\frac{V_{p}}{V_{s}}$ (between 1.6 and 1.8) only change the first term on the right-hand side $(C)$ between -0.22 and -0.1 , producing slight shifts in the corresponding linear increase. The crossed dark-gray lines in Fig. 4a show Eq. (1) for $\frac{V_{p}}{V_{s}}=1.7(C=-0.15)$, which correctly underlies the logarithmic trend of $t_{r}$. Our selection criterion is to discard data corresponding to $\frac{V_{p}}{V_{s}}$ lower than 1.65 , as these could not be reliably related to $S$ wave scattering.

The black, thin, continuous lines and the coefficients $\left(A_{r}\right.$ and $\left.B_{r}\right)$ in each panel of Fig. 4a show the theoretical log-log increase of peak-delays $\left(\log _{10}\left(t_{r}^{T}(f)\right)\right)$ with respect to $\log _{10}\left(t_{p}\right)$ (ordinate axis) at different frequencies (different panels). This is the result of the power law fit [13]:

$$
\log _{10}\left(t_{r}^{T}(f)\right)=A_{r}(f)+B_{r}(f) \log _{10}\left(t_{p}\right) .
$$

The variations with respect to these trends $\left(\Delta \log _{10}\left(t_{r}\right)\right)$, given by:

$$
\Delta \log _{10}\left(t_{r}(f)\right)=\log _{10}\left(t_{r}(f)\right)-\log _{10}\left(t_{r}^{T}(f)\right),
$$

are interpreted as the relative strength of accumulated $S$ wave scattering along each ray path. $t_{r}$ fluctuations are therefore induced by strong nearsource/near-receiver scattering [15]: a small $\delta \log _{10}\left(t_{r}\right)$ implies either the absence of strong medium heterogeneities or strong absorption along the ray 
path, whereas a large $\delta \log _{10}\left(t_{r}\right)$ indicates that the ray path crosses a strong heterogeneity [1, 12, 3].

The coefficients $A_{r}$ and $B_{r}$ are affected by high uncertainties, comparable with the values of $\delta \log _{10}\left(t_{r}\right)$ and indicative of high scattering, particularly at a short distance from the source (Fig. 4a) as seen in other volcanic cones [14]. The correction of the travel-time dependence with a linear fit is therefore imprecise, as indicated by the dispersion of $\log _{10}\left(t_{r}\right)$ measurements and the corresponding low coefficient of determination relative to the fits $\left(R^{2}\right)$, obtained from the residual variance from a fitted model (Fig. 4a). These shortcomings forbid to give valence to absolute $\Delta \log _{10}\left(t_{r}\right)$ values and will be considered in our interpretation. Nevertheless, the increasing quality of the linear fit with frequency is analogous to what is observed at regional scale [3]. Since changes in the velocity ratios cannot explain such high peak-delay variations we safely assume that $t_{r}$ is a measurement of localized $S$ wave heterogeneity.

We measure peak-delays along each 3D ray and assign its value to each $2 \mathrm{~km}$ side block crossed by the ray. Then we obtain the values in each $2 \mathrm{D}$ rectangle by averaging the measurements with the same lateral location obtained at different depths. In the final maps (Fig. 5a) the measurements are interpolated at each $2 \mathrm{D}$ node crossed by at least two rays with those of the eight nearest neighbors. The processes of depth-averaging and interpolation reduce the absolute variations of the peak-delay measurements. 


\subsection{Inverse coda quality factor mapping}

We use the decay of the energy envelope with lapse-time $t$ from the origin time of the earthquake to measure the coda quality factor $Q_{c}$ :

$$
E(t, f)=S(f) t^{-1.5} \exp -\left(\frac{2 \pi f t}{Q_{c}(f)}\right) .
$$

The power spectral energy density $E(t, f)$ at frequency $f$ is dependent on both a frequency-dependent source/site term $(S(f))$ and a frequency-dependent $Q_{c}^{-1}(f)$. In each frequency band, $Q_{c}^{-1}(f)$ is obtained from the least-square linear fit of $E(t, f) t^{1.5}$ as a function of $t$. We describe the $Q_{c}^{-1}(f)$ dependency on lapse-time by using the starting time $\left(t_{W}\right)$ and the length $\left(L_{W}\right)$ of the window used to measure it (Fig. 3), in analogy with Calvet et al. [3]. We choose these two parameters in order to obtain a quantity that is as dependent on absorption as possible, reducing the effect of scattering anisotropy.

Calvet and Margerin [16] in fact prove that the anisotropy of scattering tunes $Q_{c}$ measurements at short lapse-times and high frequencies, while the temporal decay in the late coda of crustal seismograms is mostly sensitive to absorption. In media of higher heterogeneity coda waves at short lapse-times may be affected by other coherent-like effects induced for example by topography $[14,17,8]$. However, even if the impact of anisotropy on $Q_{c}$ is generally complex, in volcanic media the quasi-isotropic scattering approximation fits seismic intensity observations at late lapse-times well $[18,2]$. In a volcano either multiple-scattering or diffusion act on the seismic wave fields, therefore we may reasonably expect $Q_{c}$ to be mainly influenced by absorption at late lapse-times $[14,2]$.

We observe smaller dependence of $Q_{c}$ on window length if we measure it using time-windows larger than $L_{W}=10 \mathrm{~s}$. The maximum available lapse- 
time to obtain stable $Q_{c}$ measurements is $t_{W}=25 \mathrm{~s}$ due to the decrease in signal-to-noise ratio. $Q_{c}$ is therefore measured from each envelope decrease over a window of length $L_{W}=10 \mathrm{~s}$ starting at $t_{W}=25 \mathrm{~s}$ (Fig. 3). The values of $Q_{c}^{-1}(f)$ measured in this time window are accepted and mapped when the signal-to-noise ratio is greater than 4 and the correlation coefficient of the linear regression is greater than 0.5. We obtain on average 360 measurements at each frequency.

We define the most probable values of $Q_{c}^{-1}(f)$ as lying between the 10th and 90th percentiles of their statistical distribution (Fig. 4b). The strong skewness of the $Q_{c}^{-1}(f)$ measurements is typical of lognormal positive random variables, but the different shape factors and skewness at different frequencies (shown on each panel) are clearly related to important variations in the medium properties. There are basically three possible explanations for the differences in statistical distribution at different frequencies, all related to coda composition/sensitivities:

1. the increased resolution and corresponding specific depth sampling of the measurements with increasing frequencies,

2. the difference in coda-intensity composition, with surface waves components decreasing with increasing frequency with respect to $S$ bodywave components [19, 20],

3. following Jing et al. [19], that absorption dominates over scattering at higher frequencies only $(12-18 \mathrm{~Hz})$.

We finally map the relative variations of the $Q_{c}^{-1}(f)$ measurements with respect to their average $Q_{m}^{-1}(f)\left(Q_{m} / Q_{c}\right.$, Fig. 3b), where $Q_{m} / Q_{c}>1$ (red colors) indicates high absorption. The mapping approach is the same applied 
to peak-delays. In addition, we provide an estimate of sensitivity and reliability of all the 2D maps showing the ray-dependent average depth (Fig. 5c) and hit count (Fig. 5d), respectively. The comparison between the depthmap and Fig. 5a,b is just indicative, as different frequencies correspond to different lateral and in-depth sensitivities for both parameters.

Theoretical and computational coda-generation models as well as array analysis measurements indicate that volcanic coda waves are composed of a complex mixture of surface and body waves. This mixture changes with frequency, affecting the depth sensitivity of coda-related observations [14, 19, 20, 4]. At Mount St. Helens the topography of the volcanic edifice and the high-contrast materials in the feeding system and surroundings may cause quick direct-wave decoherence and low-frequency rise of surface/guided waves in the coda $[21,14,8]$. We will take into account these effects in the interpretation of the results, which is dependent on the effective lateral- and depth-sensitivity of coda waves at different frequencies [19, 20, 4].

\subsection{Statistical analyses and parameter space separation.}

Our study relies on the assumption that $t_{r}$ and $Q_{c}$ are estimators of different attenuation mechanisms. To test this assumption we perform both a principal component and a correlation analysis to estimate the relative dependence between the two quantities. A principal component analysis (PCA) rotates data into eigenvalue space, with size-ordered orthogonal (independent) axes of data variability, whose orientation in the original data space is expressed in the contributions of the original variable axes to each new principal component axis. Since we only have two axes per frequency, we provide a preliminary study of the correlation of all measurements at sin- 
gle data points. We also consider possible correlations at different frequencies. The results (Table 1) show (1) that both $S$ wave measurements are positively correlated with their immediate lower-frequencies but only peakdelay time correlations are relevant at all frequencies (larger than 0.6), (2) the two measurements are uncorrelated in each frequency band, and (3) $\varepsilon$ and the peak-delay times are uncorrelated. This last observation hints at a strong difference in sampling/nature between $P$ wave phases and either $S$ or surface-scattered amplitudes.

The percent variabilities explained by the first principal component at each frequency obtained by a standard PCA do not yield additional information. In Fig. 6a we show the scores at $3 \mathrm{~Hz}$, where it is apparent that (1) we have no outliers that can be efficiently interpreted and (2) the PCA hardly provides any improvement on our results. On the other hand, by considering the 9 measurements at each block jointly (4 frequencies for peak-delays and $Q_{c}$ each plus $\varepsilon$ ) we obtain more information on the relative contribution of each measurement to the general plot. Fig. $6 \mathrm{~b}$ shows the eigenvalues of the correlation matrix in this case. The first two (three) components contribute most to data variability, explaining $62.5 \%$ (74.8\%) of overall data variability. The main contributing original variables split neatly along the expected classification: the first principal component (PC1) corresponds to the peak-delay times, while the second (PC2) is the opposite of the inverse coda quality factors. This relationship is evident by comparing the patterns in Fig. 5a,b at $3 \mathrm{~Hz}$ (large scale), which are generally anti-correlated. These results indicate strong variations in the nature, scale, and depth sampling of the $Q_{c}^{-1}(f)$ measurements at different frequencies as well as that the two 
measurements under study are complementary.

Our interpretation is performed after separating the measurements depending on their quadrant in the parameter space (Fig. 7a). In Fig. 7b we show areas marked by green (low scattering and absorption), orange (higher $Q_{c}^{-1}(f) /$ absorption), cyan (higher $\Delta \log _{10}\left(t_{r}\right) /$ scattering), and red (high scattering and absorption). To test the reliability of this approach we also apply a K-means cluster analysis with Euclidean distance and the elbow method (Fig. 8a) using the approach described by De Siena et al. [10]. This technique provides a separate quantitative interpretation to our measurements, obtained by discussing for each panel in Fig. 8b the relative position of the clusters and by allocating it in space (Fig 8c). The elbow method indicates that either two or three clusters can be chosen before the plateau in reduction (Fig. 8a, the reduction corresponding to three clusters is marked by the dotted gray line), dependent on the percentage of centroid reduction we want to achieve. By choosing three clusters the centroids are distributed along the same pattern at all frequencies (Fig. 8b), strongly depending on the $Q_{m} / Q_{c}$ variations with respect to the mean. The cyan cluster marks regions of lower $Q_{m} / Q_{c}$ and generally higher $\delta \log _{10}\left(t_{r}\right)$, whereas the orange cluster has opposite characteristics. The average measurements are characterized by the gray cluster. We have to define at least 4 clusters to reach a $90 \%$ reduction (marked by the crossed gray line in Fig. 8a). In addition, the analysis gives a certain prominence to the $Q_{c}$ measurements over peak-delay times. Therefore, the cluster analysis assumes sharp divisions in our measurements, which are not completely justified by the reduction in point-to-centroid distance, but can still be used to check the most relevant results obtained by using a 
parameter space separation (Fig. 7b).

\section{Results and discussion}

At all frequencies the southwestern $S$ wave high-scattering anomaly (Fig. 5a) matches almost perfectly with the area of maximum small-scale (average dimension $3 \mathrm{~km}$ ) geological [22], magnetic [23], and in-depth $P$ wave velocity heterogeneity (Fig. 2a - see also Lees and Crosson [24]). The same correlation between high $P$ wave and $S$ wave scattering does not characterize the second south-north trending high $P$ wave heterogeneity anomaly in Fig. 2a, marking the northern flank of the volcanic edifice. We infer that these results are due to differences in the properties of the material comprising these two regions. Scattering and absorption maps (Figs. 5a,b) are seemingly anti-correlated at low frequencies, with the $Q_{c}^{-1}$ maps showing major changes in pattern locations with increasing frequencies. We remark in particular how at $6 \mathrm{~Hz}$ the contrast between high and low $Q_{c}^{-1}(f)$ follows the St. Helens Seismic Zone (SHZ), which appears as an interface separating tectonic units of different nature/scale [24]. With increasing frequency high peak delays and low $Q_{c}$ patterns result increasingly correlated with each other, as reported by the results of the correlation analysis (Table 1). In particular, cluster analysis confirms that the SHZ represents a clear geological and tectonic interface, separating rocks of different nature in the upper panels (Fig 8c, $3 \mathrm{~Hz}$ and 6 $\mathrm{Hz}$ ). Also, with increasing frequencies, this trend rotates, running approximately southwest to northeast across the entire region (Figs 8c, $12 \mathrm{~Hz}$ and $18 \mathrm{~Hz}$ ). 


\subsection{Low frequencies: debris flows, geological units, and tectonic trends}

Both high $\Delta \log _{10}\left(t_{r}\right)$ and high $Q_{m} / Q_{c}($ red, Fig. 7a) are typical at continental scale and in experimental studies when seismic waves traverse media with a high concentration of melt phases and fluids $[25,1,5,7,12]$. In our setting high-scattering anomalies, if mirrored by high-absorption anomalies, are possibly caused by unconsolidated rocks, sediments, magma, and fluids. The continuous red patterns in Fig. 7b actually cross the cone at all frequencies, either related to surface geology or providing important information on the location of the main feeding systems. In addition, the relative positions of the anomalies marked by the other colors provide important insight into the main tectonic trends and structures characterizing the area.

The only relevant high-scattering and high-absorption anomaly (red) at $3 \mathrm{~Hz}$ (Fig. 7b) follows a northwest-to-southeast trend, covering the northern and eastern flanks of the volcano. The northern part of the anomaly follows the largest section of the meltwater debris flows following the collapse of the northern flank of the volcano in 1980. The contour of the visible part of these partially buried flows between 1980 and 1983 [26] are delineated in Figs. S1-S2 (see Supplementary Information) by using geographical information systems, satellite imagery, and digital elevation models of USGS data with the method described by Ernst et al. [27]. On the other hand, the eastern flank of the volcanic edifice is not intersected by any visible debris flow, the corresponding red anomaly possibly being the expression of deeper highscattering and high-absorption structures.

In Fig. $7 \mathrm{c}$ we report the resolvable features (minimum dimension $=2$ $\mathrm{km}$ ) of the visible debris flows in 1983: this spatial correlation suggests an 
unexpected sensitivity of our method to the shallowest layers of the crust (i=200 m). Low-frequency $Q_{c}$ measurements sample shallower structures than those expected from the average ray-dependent depth sampling. At $3 \mathrm{~Hz}$, this may be explained by the strong influence of surface waves on low-frequency coda-waves $[19,20]$.

The cyan/green regions west of the SHZ depict a sharp north-south contrast with the orange regions east of it (Fig. 7b-c, $3 \mathrm{~Hz}$ ). Such a contrast consistently matches the difference in tectonic composition/lithology reported by Finn and Williams [23] and Evarts et al. [22] by using magnetic anomalies and surface geology, respectively. In particular, the orange region maps a large high magnetic/high velocity anomaly, impervious to fluid injection, comprising the Spirit Lake Pluton, and running east of MSH and Marble Mt. (Fig. 7b-c, $3 \mathrm{~Hz}$, the contour is shown by white/orange dotted lines east of the SHZ).

The second significant (although smaller) high magnetic anomaly (whitedotted line west of the SHZ, Fig. 7b-c, $3 \mathrm{~Hz}$ ), which also corresponds to a Pluton (Fig. 7c, Spud Mount, see Finn and Williams [23]), shows both low $Q_{m} / Q_{c}$ and low peak-delay times. This difference may only be explained by either a lateral insensitivity of coda waves to structures (wavelengths) smaller than $10 \mathrm{~km}$ or a greater lateral and in-depth sampling of tectonic units at low frequencies. While the first interpretation would not justify the precise contour obtained for the debris flows, the last hypothesis is supported by rotation of the high scattering/high absorption contrast, which coincides at $6 \mathrm{~Hz}$ with the trace of the SHZ (Fig. 7c, dotted cyan and orange lines). The SHZ evidently separates two media with different scattering/absorption 
properties and fracturing.

In our interpretation, these results show the effect of two important tectonic structures. At depths greater than $10 \mathrm{~km}$ the high absorption east of the SHZ could be induced by large deep melt phases, possibly a direct image of the highly conducting Southern Washington Cascades Conductor hypothesized by electromagnetic studies [28, 29]. Nevertheless, both high $\Delta \log _{10}\left(t_{r}\right)$ and high $Q_{m} / Q_{c}$ should mark the presence of melt and fluids $[25,1,5,7,12]$, while only different $Q_{m} / Q_{c}$ characteristics separate the two regions (Fig. 7b, $3 \mathrm{~Hz}$ ). We infer that the cyan and orange anomalies are more likely to depict the transition between the low-velocity and lower scattering Cascade Arc Crust (orange, north and east of the SHZ, Fig. 7c, $6 \mathrm{~Hz}$ ) and the high-velocity Siletz Terrane (cyan/green, south and west of the SHZ, see e.g. Parsons et al. [30]), whose top may strongly contribute to late coda intensities.

The area of highest $S$ wave scattering is always located west southwest of the SHZ (cyan Figs. 7b-c, $3 \mathrm{~Hz}$, also compare Fig 5a). In Fig. 7b, at $3 \mathrm{~Hz}$, we impose a black dashed line on this area, showing both the contour of the strongest in-depth velocity contrasts between the surface and 9 $\mathrm{km}[24]$ and the highest small-scale (average dimension $3 \mathrm{~km}$ ) surface and magnetic heterogeneity [22, 23]. Two travel-time tomography studies show different velocity characteristics at depths greater than $6 \mathrm{~km}$ in this aseismic region, with either low [31] or high velocities [9]. While the strong in-depth and lateral contrasts and the high scattering provide important evidence of past volcanic activity, we infer no large upper crustal emplacement of either magma or hot fluids in the region before the eruption, due to its low 
absorption characteristics at all frequencies [31].

At $6 \mathrm{~Hz}$, a high-scattering and high-absorption region crosses the volcanic edifice along the SHZ trend, from its northwestern to its southeastern flank (Fig. 7b, red). Coda waves may be partially comprised of surface waves in this frequency range, with the maps influenced by unconsolidated superficial structures. Nevertheless, the red anomaly shows a trend depicted in the $P$ wave travel-time tomography of Waite and Moran [9] by low-velocity anomalies at a depth of $2.9 \mathrm{~km}$ (Fig. 7b-c, $6 \mathrm{~Hz}$, white/red dashed contour lines). Also, the trend fades in its northern part with respect to $3 \mathrm{~Hz}$. Considering the uncertainties on depth sampling and coda composition affecting our data we may safely attribute the anomaly to the effect of magmatic/fluid paths located under the volcanic edifice between depths of 2 and $4 \mathrm{~km}[31,9]$.

\subsection{High frequencies: tectonic trends and feeding paths}

We expect a strong influence of body waves on the high-frequency coda intensities used to measure scattering and absorption [20]. Therefore, the analysis of the high-frequency measurements should better depict structures at the average ray-dependent depths (between depths of $2 \mathrm{~km}$ and $8 \mathrm{~km}$, Fig. 3c). In Fig. 7b, 12-18 Hz, we contour with a black dotted line the areas of highest $P$ wave velocity between depths of 4 and $6 \mathrm{~km}$ as shown by Lees and Crosson [24]. A large red anomaly marks the SHZ trend, the volcanic edifice, and reaches the southern flank of the volcano at $12 \mathrm{~Hz}$ (Fig 7b-c). The NNW-SSE trend shows persistent high-scattering and high-absorption characteristics at different frequencies (Fig. 7b, 3-12 Hz). Considering wave field distortion produced by the collapsed cone on the observed quantities $[32,17]$ this result mirrors the major role this zone of tectonic interaction 
has on the upward movement of melt phases and fluids at MSH.

In Fig. $7 \mathrm{~b}, 12 \mathrm{~Hz}$, the extension of the trend to the southern edge of the volcanic edifice is in excellent spatial correlation with a negative aeromagnetic anomaly, which marks the trend of prominent mapped contacts crossing the MSH cone [23]. Indeed, the regional structural high-scattering and high-absorption trend is possibly a fault spreading in its southwestern limit (Fig. 7c, 12), which would explain the more diffuse seismicity and less well defined seismic velocity anomalies to the south of MSH, in agreement with the interpretation of previous studies [22, 33, 24, 34]. Also, between depths of 4 and $10 \mathrm{~km}$, tertiary marine sediments may be preserved in the graben-like cracked volume west of the SHZ, increasing both scattering and absorption $[34,8]$.

With increasing frequency, the maps show a dramatic change in the relative position of the orange, cyan, and green anomalies (Fig. 7b). With respect to $6 \mathrm{~Hz}$, the high-frequency plots show a rotation of the scattering/absorption contrasts around an axis centered on the volcano from NNWSSE in a SW-NE direction (Fig 7b-c, compare $6 \mathrm{~Hz}-18 \mathrm{~Hz}$ ). In particular, at $12 \mathrm{~Hz}$ the orange region runs parallel to the NNW-SSE trend, at the end of the red trend. At $18 \mathrm{~Hz}$ the cyan anomaly splits the orange trend in two, while the red anomalies have their principal axes disposed along the SW-NE direction (red, Fig. 7b-c, $18 \mathrm{~Hz}$ ).

The SW-NE direction coincides with the strike of the steeply dipping fault, inferred between depths of 5.5 and $10 \mathrm{~km}$ by Musumeci et al. [35], and considered the cause of magma injection from depths greater than $6 \mathrm{~km}$. Total $P$ wave attenuation measurements obtained using passive data recorded 
before the 2004 eruption show a drastic decrease of attenuation below $5.5 \mathrm{~km}$ [36]. In the interpretation of Tusa et al. [36] this is due to heated rocks in proximity of the conduit, which is located only above this depth. The conduit has been recently imaged as a short vertical lineament between depths of 0 and $5 \mathrm{~km}$ under the central crater using microseismicity located with a novel large-N geophone array [37]. Therefore, the scattering/absorption contrasts at $18 \mathrm{~Hz}$, which comprise high-scattering and high-absorption SWNE anomalies, support our current understanding of magma recharge into the feeding structures of the volcano below $5 \mathrm{~km}$. In addition, the patterns hint at a crustal trend possibly related to the SW-NE greater-scale magmatic arc structure, recently placed at $9 \mathrm{~km}$ depth by Obrebski et al. [38]. These results all agree upon a strong interconnection between tectonic and feeding systems at MSH, with tectonic units driving and constraining the supply of magmatic and fluid phases to volcanic eruptions.

The smaller-scale red anomalies are the expression of either small-scale reflectors or errors in our measurements, as mirrored by the result of the elbow method at $18 \mathrm{~Hz}$ (Fig 8a): we need a strong increase in the number of clusters to reduce the model-to-data variance in this frequency band. On the other hand, the 11 most relevant variations in the parameter space (Fig. 7a, $18 \mathrm{~Hz}$, black-contoured red dotted-line) appear as a singular SW-NE trending anomaly located under the eastern and south-eastern flanks of the volcano (Fig. 7c, black-contoured red dotted-line). These regions (1) are those characterized by extreme velocity contrasts between depths of 2 and $14 \mathrm{~km},(2)$ show high conductance [29] between depths of 0 and $10 \mathrm{~km},(3)$ overlay deep low-velocity anomalies, attributed to the presence of magma [31], and (4) 
coincide with the location of a 10-to-14 km deep low-scattering anomaly, interpreted as a network of interconnected water-rich melt absorbing fractures [8]. From their $S$ wave high-absorption and high-scattering characteristics at all frequencies we conclude that the eastern and southeastern flanks of MSH are the most feasible locations of feeding paths below depths of $4 \mathrm{~km}$, at least before its 2004 explosive eruption (Fig. 7c, $18 \mathrm{~Hz}$ ).

\section{Conclusions}

The joint analysis of low-frequency 2D scattering- and absorption-dependent measurements depicts the interface between rocks of significantly varying properties respectively west and east of Mount St. Helens. It also provides an exact contour of the unconsolidated high-scattering and high-absorption debris flows following the 1980 explosive eruption, hinting at a high sensitivity of low-frequency coda waves to the first few layers of the crust. Our results depict the NNW-to-SSE oriented SHZ as a boundary between different tectonic structures having a crucial role in driving and constraining feeding paths at MSH. The anomalies corresponding to high-absorption and highscattering materials cross the volcanic edifice from its north northwestern to its south southeastern flank with increasing frequency. In our interpretation they show the extension and trend of sediments (buried inside/near to the SHZ) and magma phases/fluids (under the volcano between depths of 2 and $4 \mathrm{~km})$.

By the comparison of tomograms at different frequencies we conclude that low frequency $(3-6 \mathrm{~Hz})$ coda/scattered intensities sample both the shallowest crust, probably due to the influence of surface components, and deeper 
large-scale tectonic structures, where passive seismicity is embedded. The main characteristic of the high-frequency maps is the rotation of the tectonic/feeding trends around an axis centered on the volcano from NNW-SSE in the known direction of the regional-scale magmatic arc at $9 \mathrm{~km}$ (SW-NE). The aseismic high-scattering region WSW of the volcano, depicted in previous travel-time tomography studies as either high- or low-velocity below $6 \mathrm{~km}$ depth, shows no evident high-absorption characteristics. Indeed, the highest-scattering and highest-absorption regions are instead consistently located under either the eastern and south-eastern flanks of the volcanic edifice at all frequencies, where we infer the location of the main magma/hot fluids paths in the upper crust below $4 \mathrm{~km}$.

\section{Acknowledgments}

We thank Edoardo Del Pezzo, Ludovic Margerin, Haruo Sato, Mare Yamamoto, Tatsuhiko Saito, Malcolm Hole, and Seth Moran for the valuable suggestions regarding the methodology and interpretation. Greg Waite provided the $P$ wave velocity model of MSH. An important revision of the methods was done after two blind reviews performed before submission. The suggestions of two anonymous reviewers greatly enhanced our ability of imaging structures, interpreting our results, and testing their reliability. The facilities of the IRIS Data Management System, and specifically the IRIS Data Management Center, were used for access to waveform and metadata required in this study, and provided by the Cascades Volcano Observatory - USGS. Interaction with geologists and geographers part of the Landscape Dynamics Theme of the Scottish Alliance for Geoscience, Environment and Society 
(SAGES) has been important for the interpretation of the results.

\section{References}

[1] T. Takahashi, H. Sato, T. Nishimura, K. Obara, Strong inhomogeneity beneath Quaternary volcanoes revealed from the peak delay analysis of S-wave seismograms of microearthquakes in northeastern Japan, Geophysical Journal International 168 (1) (2007) 90-99.

[2] H. Sato, M. C. Fehler, T. Maeda, Seismic Wave Propagation and Scattering in the heterogeneous Earth: Second Edition, Springer, New York, USA, 2012.

[3] M. Calvet, M. Sylvander, L. Margerin, A. Villaseñor, Spatial variations of seismic attenuation and heterogeneity in the Pyrenees: Coda Q and peak delay time analysis, Tectonophysics 608 (2013) 428-439.

[4] J. Prudencio, E. Del Pezzo, J. Ibáñez, E. Giampiccolo, D. Patané, Twodimensional seismic attenuation images of Stromboli Island using active data, Geophysical Research Letters 42 (6) (2015) 1717-1724.

[5] E. Carcolé, H. Sato, Spatial distribution of scattering loss and intrinsic absorption of short-period $\mathrm{S}$ waves in the lithosphere of Japan on the basis of the Multiple Lapse Time Window Analysis of Hi-net data, Geophysical Journal International 180 (1) (2010) 268-290.

[6] M. C. Fehler, M. Hoshiba, H. Sato, H. Obara, Separation of scattering and intrinsic attenuation for the Kanto-Tokai region, Japan, using 
mesurements of S-wave energy versus hypocentral distance, Geophysical Journal International 108 (1992) 787-800.

[7] E. Del Pezzo, F. Bianco, Two-layer earth model corrections to the MLTWA estimates of intrinsic-and scattering-attenuation obtained in a uniform half-space, Geophysical Journal International 182 (2) (2010) 949-955.

[8] L. De Siena, C. Thomas, G. P. Waite, S. C. Moran, S. Klemme, Attenuation and scattering tomography of the deep plumbing system of Mount St. Helens, Journal of Geophysical Research: Solid Earth 119 (11) (2014) 8223-8238.

[9] G. Waite, S. Moran, Vp Structure of Mount St. Helens, Washington, USA, imaged with local earthquake tomography, Journal of Volcanology and Geothermal Research 182 (2009) 113-122.

[10] L. De Siena, E. Del Pezzo, F. Bianco, A scattering image of Campi Flegrei from the autocorrelation functions of velocity tomograms, Geophysical Journal International 184 (3) (2011) 1304-1310.

[11] L. De Siena, E. Del Pezzo, C. Thomas, A. Curtis, L. Margerin, Seismic energy envelopes in volcanic media: in need of boundary conditions, Geophysical Journal International 192 (1) (2013) 326-345.

[12] J. N. Tripathi, M. Sato, M. Yamamoto, Envelope broadening characteristics of crustal earthquakes in northeastern Honshu, Japan, Geophysical Journal International 182 (2) (2010) 988-1000. 
[13] T. Saito, H. Sato, M. Ohtake, Envelope broadening of spherically outgoing waves in three-dimensional random media having power law spectra, Journal of Geophysical Research 107 (B5) (2002) 2089-2103.

[14] U. Wegler, Analysis of Multiple Scattering at Vesuvius Volcano, Italy, using Data of the TomoVes active seismic experiment, Journal of Volcanology and Geothermal Research 128 (2003) 45-63.

[15] T. W. Chung, J. M. Lees, K. Yoshimoto, E. Fujita, M. Ukawa, Intrinsic and scattering attenuation of the Mt Fuji Region, Japan, Geophysical Journal International 177 (3) (2009) 1366-1382.

[16] M. Calvet, L. Margerin, Lapse time dependence of coda Q: anisotropic multiple-scattering models and application to Pyrenees, Bulletin of the Seismological Society of America 103 (3) (2013) 1993-2010, doi: $10.1785 / 0120120239$.

[17] Y. Gao, N. Zhang, Scattering of cylindrical SH waves induced by a symmetrical V-shaped canyon: near-source topographic effects, Geophysical Journal International 193 (2013) 874-885.

[18] M. Yamamoto, H. Sato, Multiple scattering and mode conversion revealed by an active seismic experiment at Asama volcano, Japan, Journal of Geophysical Research 115 (B0) (2010) 7304-7317.

[19] Y. Jing, Y. Zeng, G. Lin, High-Frequency Seismogram Envelope Inversion Using a Multiple Nonisotropic Scattering Model: Application to Aftershocks of the 2008 Wells Earthquake, Bulletin of the Seismological Society of America 104 (2) (2014) 823-839. 
[20] D. Galluzzo, M. L. Rocca, L. Margerin, E. D. Pezzo, R. Scarpa, Attenuation and velocity structure from diffuse coda waves: constraints from underground array data, Physics of the Earth and Planetary Interiors .

[21] J. Neuberg, T. Pointer, Effects of volcano topography on seismic broadband waveforms, Geophysical Journal International 143 (2000) 239-248, doi:10.1046/j.1365-246x.2000.00251.xs.

[22] R. C. Evarts, R. P. Ashley, J. G. Smith, Geology of the Mount St. Helens area: Record of discontinuous volcanic and plutonic activity in the Cascade Arc of southern Washington, Journal of Geophysical Research 92 (B10) (1987) 10155-10169.

[23] C. Finn, D. L. Williams, An aeromagnetic study of Mount St. Helens, Journal of Geophysical Research 92 (B10) (1987) 10194-10206.

[24] J. M. Lees, R. S. Crosson, Tomographic inversion for threedimensional velocity structure at Mount St. Helens using earthquake data, Journal of Geophysical Research 94 (1989) 5716-5728.

[25] D. H. Johnston, Attenuation: A state-of-the-art summary, in: M. N. Toksoz, D. H. Johnston (Eds.), Seismic Wave Attenuation, SEG reprint series No. 2, 123-139, 1981.

[26] J. J. Major, L. E. Mark, Peak flow responses to landscape disturbances caused by the cataclysmic 1980 eruption of Mount St. Helens, Washington, Geological Society of America Bulletin 118 (7-8) (2006) 938-958.

[27] G. Ernst, M. Kervyn, R. M. Teeuw, Advances in the remote sensing of volcanic activity and hazards, with special consideration to applications 
in developing countries, International Journal of Remote Sensing 29 (22) (2008) 6687-6723.

[28] G. D. Egbert, J. R. Booker, Imaging crustal structure in southwestern Washington with smalll magnetometer arrays., Journal of Geophysical Research 98 (1993) 15967-15985.

[29] G. Hill, T. Caldwell, W. Heise, D. Chertkoff, H. Bibby, M. Burgess, J. Cull, R. Cas, Distribution of melt beneath Mount St Helens and Mount Adams inferred from magnetotelluric data, Nature Geoscience 2 (11) (2009) 785-789.

[30] T. Parsons, R. J. Blakely, T. M. Brocher, N. I. Christensen, M. A. Fisher, E. Flueh, F. Kilbride, J. H. L. aand K. Miller, U. S. ten Brink, A. M. Trehu, R. E. Wells, Crustal Structure of the Cascadia Fore Arc of Washington, in: R. Kayen (Ed.), Earthquake Hazards of the Pacific Northwest Costal and Marine Regions, USGS Professional Paper, 1661D, 2005.

[31] J. Lees, The magma system of Mount St. Helens: non-linear highresolution P-wave tomography, Journal of Volcanology and Geothermal Research 53 (1) (1992) 103-116.

[32] J. F. Anderson, J. M. Lees, G. P. Waite, J. B. Johnson, Source and Propagation Effects on Near-Field Co-Eruptive Ground Motion at Santiaguito Volcano, Guatemala, Bulletin of the Seismological Society of America 102 (2) (2012) 696-706. 
[33] W. D. Stanley, C. Finn, J. L. Plesha, Tectonics and conductivity structures in the southern Washington Cascades, Journal of Geophysical Research 92 (B10) (1987) 10179-10.

[34] S. C. Moran, J. M. Lees, S. Malone, P-wave crustal velocity structure in the greater Mount Rainier area from local earthquake tomography, Journal of Geophysical Research 104 (B5) (1999) 10775-10786.

[35] C. Musumeci, S. Gresta, S. D. Malone, Magma system recharge of Mount St. Helens from precise relative hypocenter location of microearthquakes, Journal of Geophysical Research 107 (B10) (2002) 2264.

[36] G. Tusa, S. D. Malone, E. Giampiccolo, S. Gresta, C. Musumeci, Attenuation of Short-Period P Waves at Mount St. Helens, Bulletin of the Seismological Society of America 94 (4) (2004) 1441-1455, doi: $10.1785 / 012003040$.

[37] S. M. Hansen, B. Schmandt, Automated detection and location of microseismicity at Mount St. Helens with a large-N geophone array, Geophysical Research Letters 42 (18) (2015) 7390-7397.

[38] M. Obrebski, G. A. Abers, A. Foster, Magmatic arc structure around Mount Rainier, WA, from the joint inversion of receiver functions and surface wave dispersion, Geochemistry, Geophysics, Geosystems 16 (1) (2015) 178-194. 
${ }_{626}$ Author contributions. L.D.S., M.C. and C.T. wrote the paper. L.D.S., M. C., ${ }_{627}$ K. J. W. and A. R. T. J. carried out the modeling. All authors contributed ${ }_{628}$ to the interpretation and manuscript editing.

629 Additional information. Supplementary information accompanies this paper. ${ }_{630}$ Correspondence and requests for materials should be addressed to L.D.S. 


\begin{tabular}{l|c|c|c|c|c|c|c|c|c}
\hline & $\delta \log _{10}\left(t_{r}\right), 3 \mathrm{~Hz}$ & $6 \mathrm{~Hz}$ & $12 \mathrm{~Hz}$ & $18 \mathrm{~Hz}$ & $Q_{m} / Q_{c}, 3 \mathrm{~Hz}$ & $6 \mathrm{~Hz}$ & $12 \mathrm{~Hz}$ & $18 \mathrm{~Hz}$ & $\varepsilon$ \\
\hline$\delta \log _{10}\left(t_{r}\right), 3 \mathrm{~Hz}$ & 1 & & & & & & & & \\
\hline $6 \mathrm{~Hz}$ & 0.776 & 1 & & & & & & & \\
\hline $12 \mathrm{~Hz}$ & 0.773 & 0.866 & 1 & & & & & & \\
\hline $18 \mathrm{~Hz}$ & 0.741 & 0.842 & 0.958 & 1 & & & & & \\
\hline$Q_{m} / Q_{c}, 3 \mathrm{~Hz}$ & 0.161 & 0.015 & 0.078 & -0.019 & 1 & & & & \\
\hline $6 \mathrm{~Hz}$ & -0.120 & -0.230 & -0.214 & -0.290 & 0.341 & 1 & & & \\
\hline $12 \mathrm{~Hz}$ & 0.135 & -0.033 & -0.013 & -0.065 & 0.229 & 0.451 & 1 & & \\
\hline $18 \mathrm{~Hz}$ & 0.162 & 0.049 & 0.048 & -0.006 & 0.084 & 0.136 & 0.696 & 1 & \\
\hline$\varepsilon$ & 0.079 & 0.015 & 0.036 & 0.056 & -0.005 & -0.040 & 0.033 & 0.068 & 1 \\
\hline
\end{tabular}

Table 1: *

Table 1: The matrix shows the Pearson correlation values between $\delta \log _{10}\left(t_{r}\right), Q_{m} / Q_{c}$, and $\varepsilon$ measurements at different frequencies. Statistically significant correlations are shown in italics. 


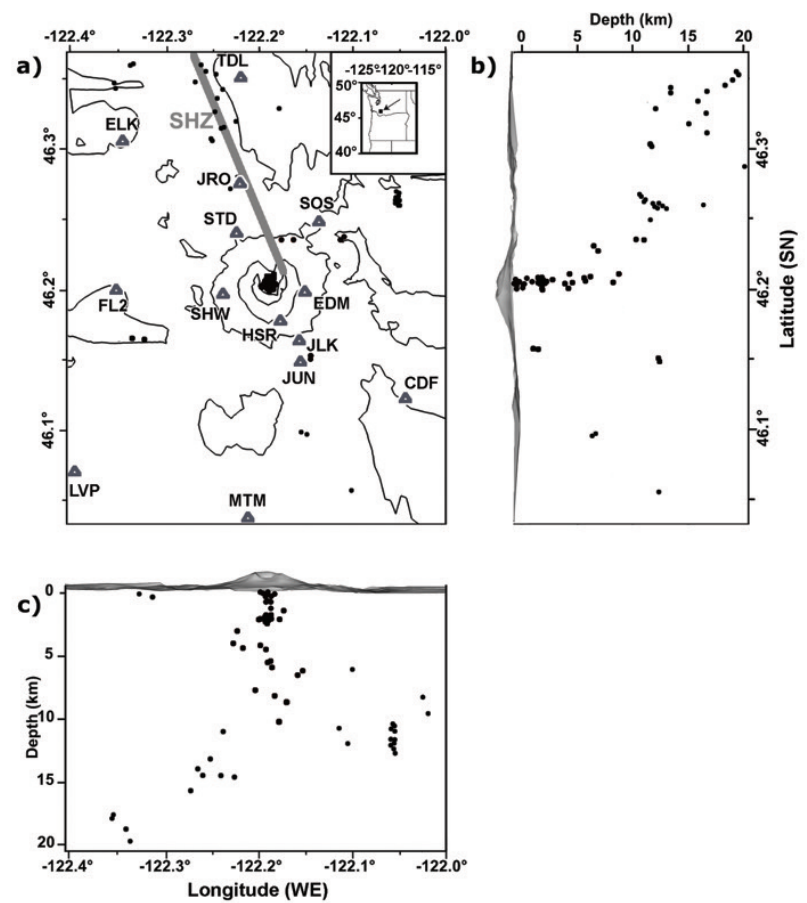

Figure 1: Seismic and geographical data. (a) A map-view plot of the permanent network stations (gray triangles and letters with white contours), recording seismicity (black circles) between 2000 and 2003 at Mount St. Helens (MSH) on topography contoured at $1 \mathrm{~km}$ intervals. The color map shows the tomographically defined 2D $P$ wave scattering $(\varepsilon)$ spatial variations. The inset (upper right corner of the map) shows the location of MSH in southern Washington. We represent the St. Helens Seismic Zone (SHZ) as a thick black line on the map. Two vertical sections running south-north (b) and west-east (c) and including topography show the projected seismicity. 


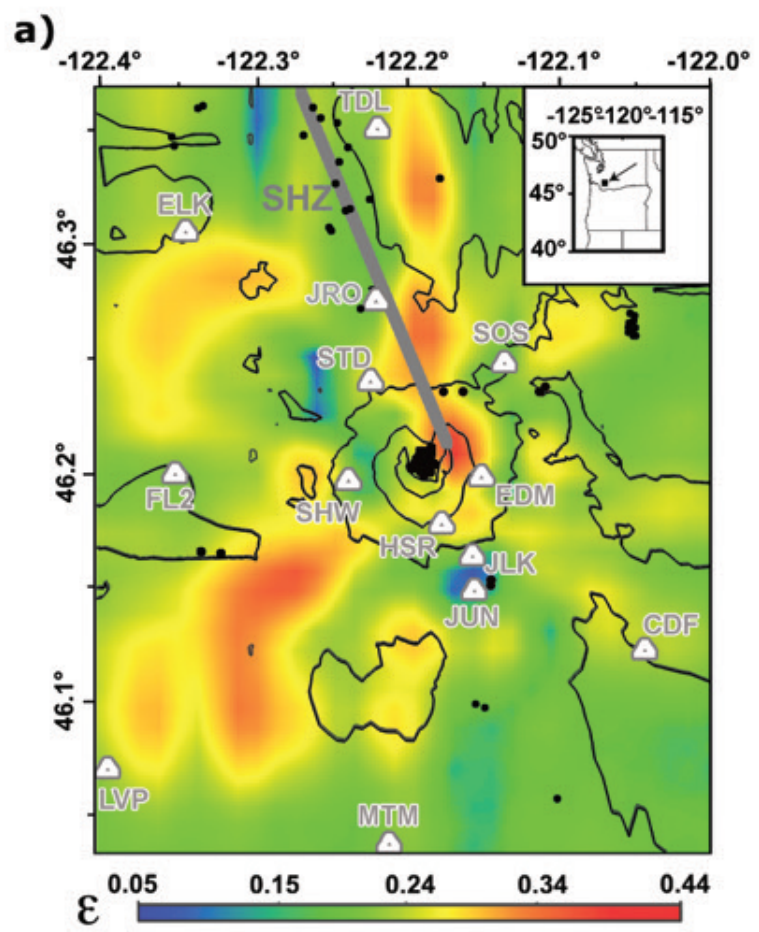

b)

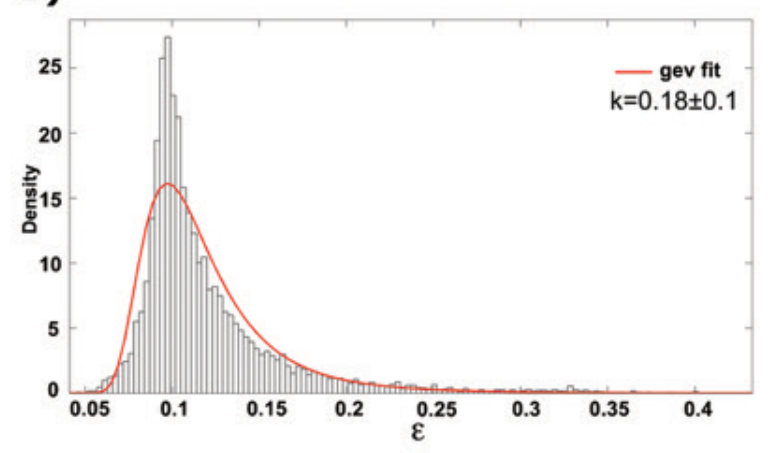

Figure 2: $P$ wave heterogeneity. (a) The color map shows the tomographically defined 2D $P$ wave scattering $(\varepsilon)$ spatial variations overlaid by the map of Fig. 1a. (b) The $\varepsilon$ data density follows a generalized extreme value distribution (gev) of second type, with shape parameter $\kappa=0.18 \pm 0.1$. 


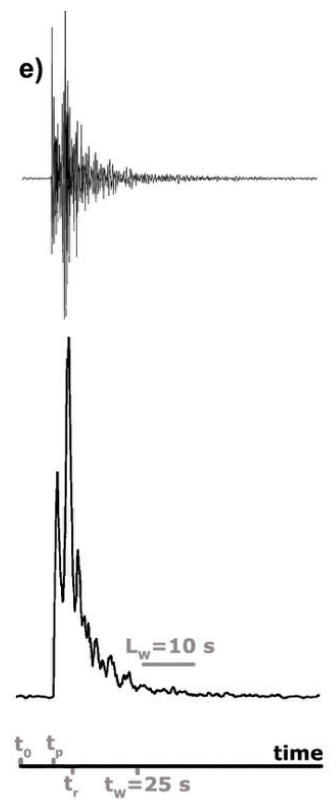

Figure 3: Seismic time measurements. An example velocity waveform and envelope with corresponding time-related quantities as defined in the study. 
a)

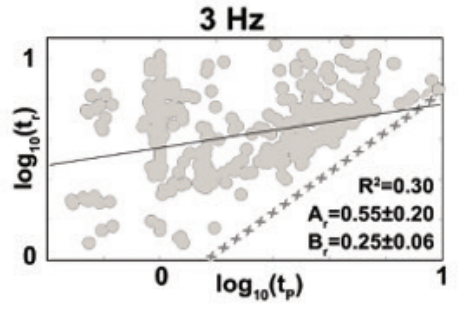

$6 \mathrm{~Hz}$

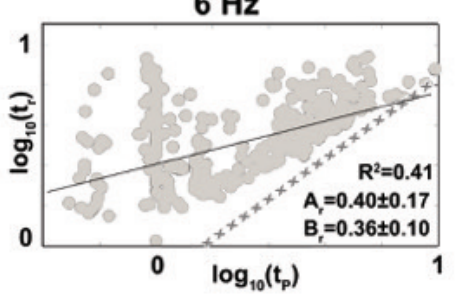

$12 \mathrm{~Hz}$

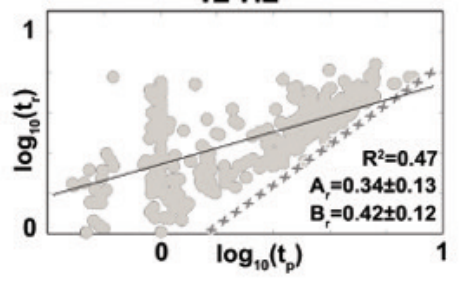

$18 \mathrm{~Hz}$

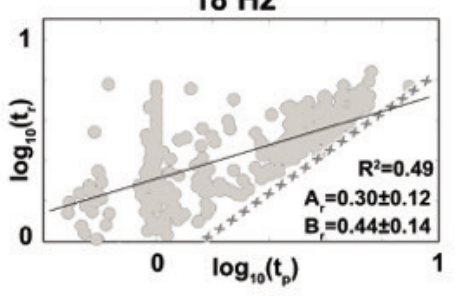

b)

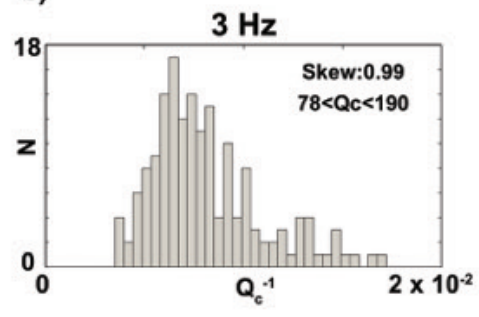

$6 \mathrm{~Hz}$

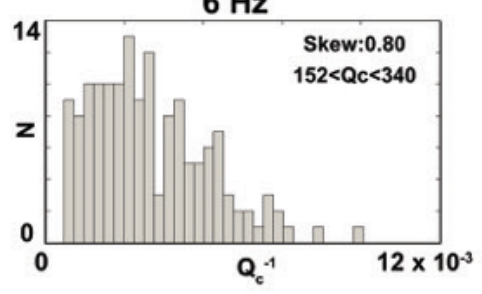

$12 \mathrm{~Hz}$

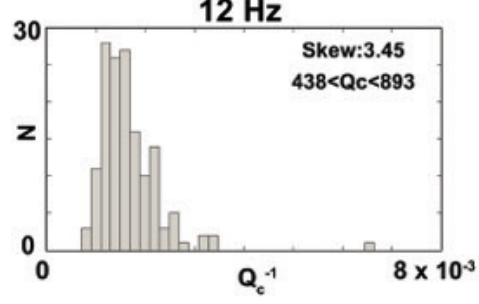

$18 \mathrm{~Hz}$

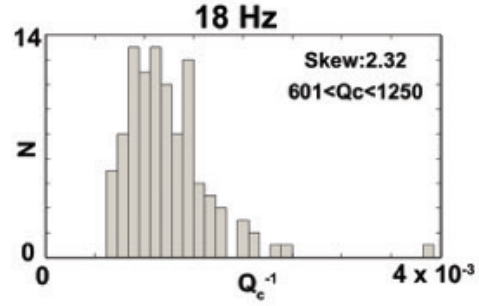

Figure 4: Peak delay fit and $Q_{c}$ statistics. (a) Logarithmic plots of the peak-delay times $\left(t_{r}\right.$, gray dots) versus the $P$ wave travel-times $\left(t_{p}\right)$ in four frequency bands. Each panel shows the coefficients $A_{r}(f)$ and $B_{r}(f)$ obtained by the power law fit (black continuous lines) with their uncertainties given by the covariance matrix as well as the coefficient of determination relative to the fits $\left(R^{2}\right)$. The x-dashed line shows the theoretical $S$ wave arrival corresponding to a $\frac{V_{p}}{V_{s}}=1.7$. (b) The histograms of the inverse coda quality factor measurements $\left(Q_{c}^{-1}\right)$ in the four frequency bands. We show the $90 \%$ confidence intervals of the average $Q_{c}$ in each panel. 

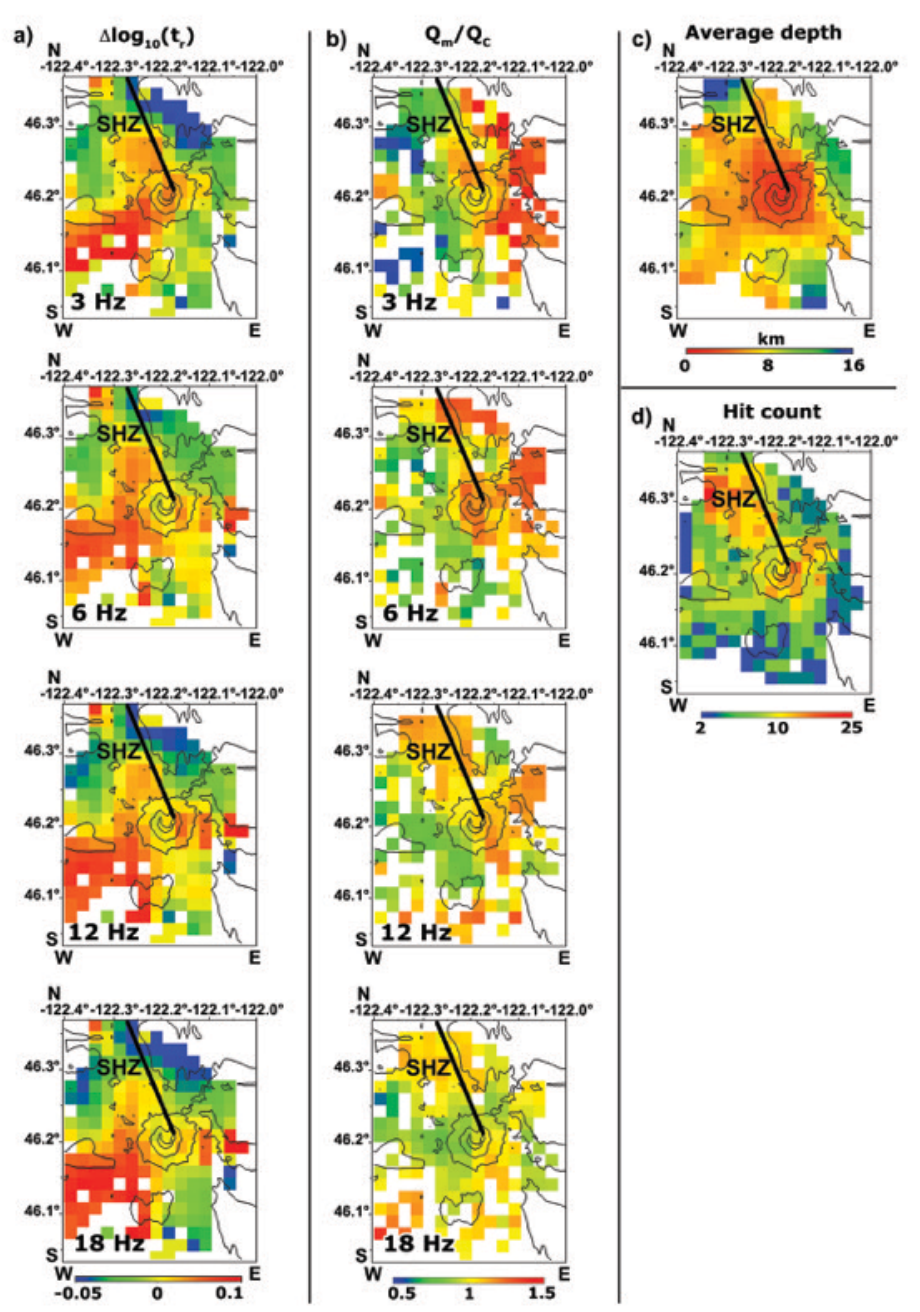

Figure 5: Peak-delay and $Q_{c}$ mapping. The contour map of Mount St. Helens is imposed on the frequency-dependent results of the $2 \mathrm{D}$ peak-delay time mapping (a) and $2 \mathrm{D} Q_{m} / Q_{c}$ mapping (b), where the square sizes are $2 \times 2 \mathrm{~km}$. (a) The color scale show the variations of the logarithm of the peak-delay times corrected for their $t_{p}$ dependency at different frequencies. (b) The color scale shows the variations of the inverse coda quality factor $\left(Q_{c}^{-1}\right)$ divided by the mean of the measurements for a 10-second long window starting at a lapse-time of $25 \mathrm{~s}\left(Q_{m}^{-1}\right)$. Average-depth map (c) and (d) hit-count are relative to the plots shown in panels (a) and (b) depending on ray geometry. We only show the squares crossed by a minimum of 2 rays. 

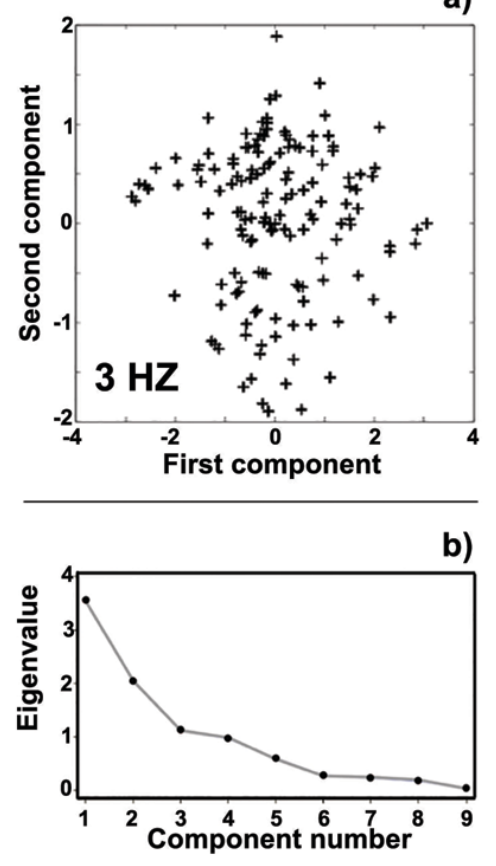

Figure 6: Results of Principal component analyses. (a) Principal component analysis scores $(+)$ in the principal component space at $3 \mathrm{~Hz}$, where the first component explains $66 \%$ of the total variability. (b) The eigenvalues of the correlation matrix obtained by considering the 9 measurements obtained in each block jointly versus the component number show the reduction in data variability. 

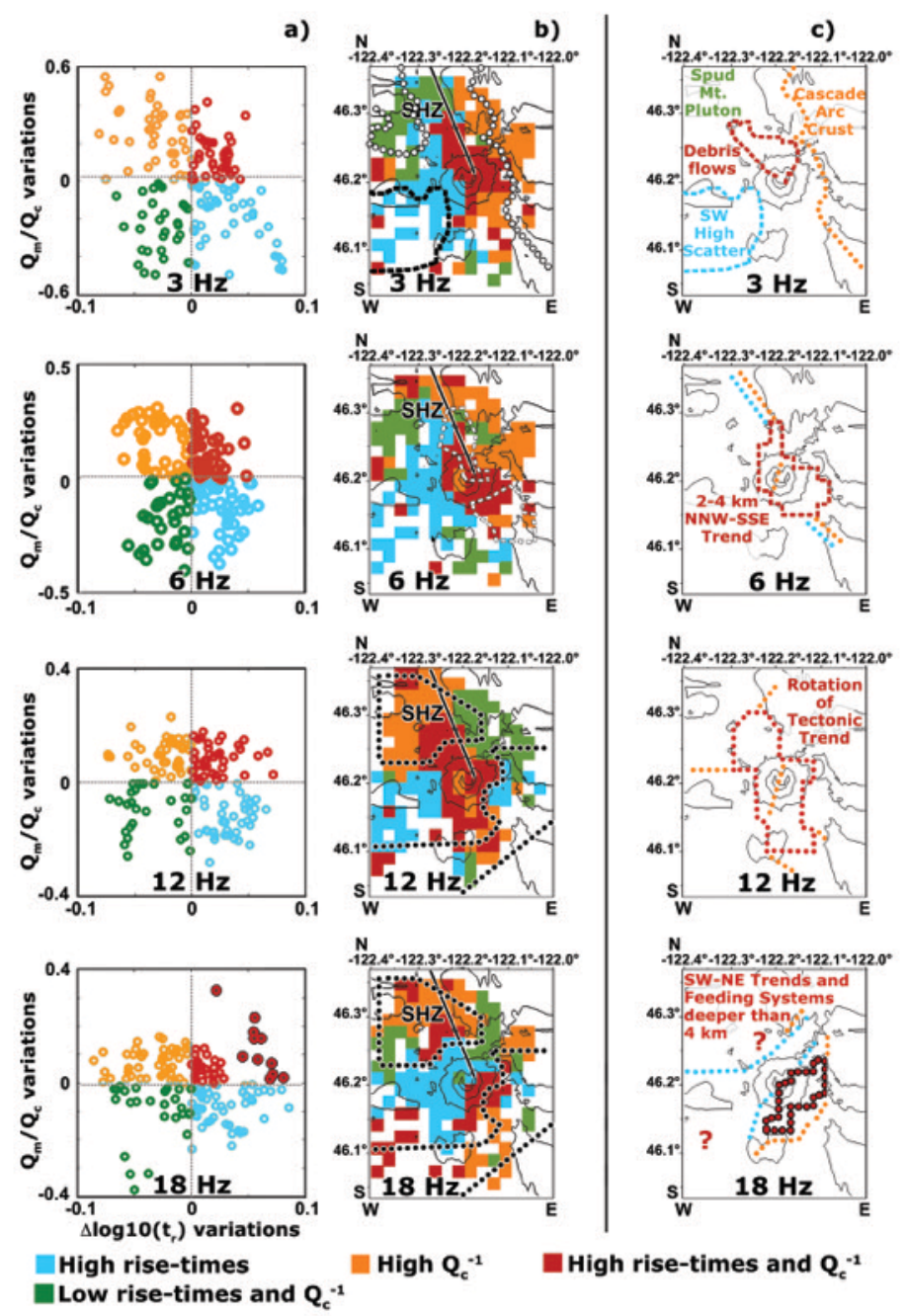

Low rise-times and $Q_{c}{ }^{-1}$

Figure 7: Mapping after separation in parameter space. (a) The 2D measurements are separated with colors in the parameter space and (b) mark scattering and absorption characteristics of the area. We contour high magnetic anomalies $(3 \mathrm{~Hz}$, white dotted curves), areas of high surface, magnetic, and depth-dependent velocity heterogeneity (3 $\mathrm{Hz}$ black dashed line), low-velocity anomalies at a depth of $2.9 \mathrm{~km}(6 \mathrm{~Hz}$, dashed white lines), and high-velocity anomalies between depths of 4 and $6 \mathrm{~km} \mathrm{(12} \mathrm{Hz} \mathrm{and} 18 \mathrm{~Hz}$, dotted black lines). c) Simplified sketches of our interpretation in different frequency bands. The red dashed line at $3 \mathrm{~Hz}$ contours debris flows larger than $2 \mathrm{~km}$ as they appear from satellite imaging. Black-contoured red dots in panels a and c $(18 \mathrm{~Hz})$ mark the areas of highest positive variation for both quantities. 


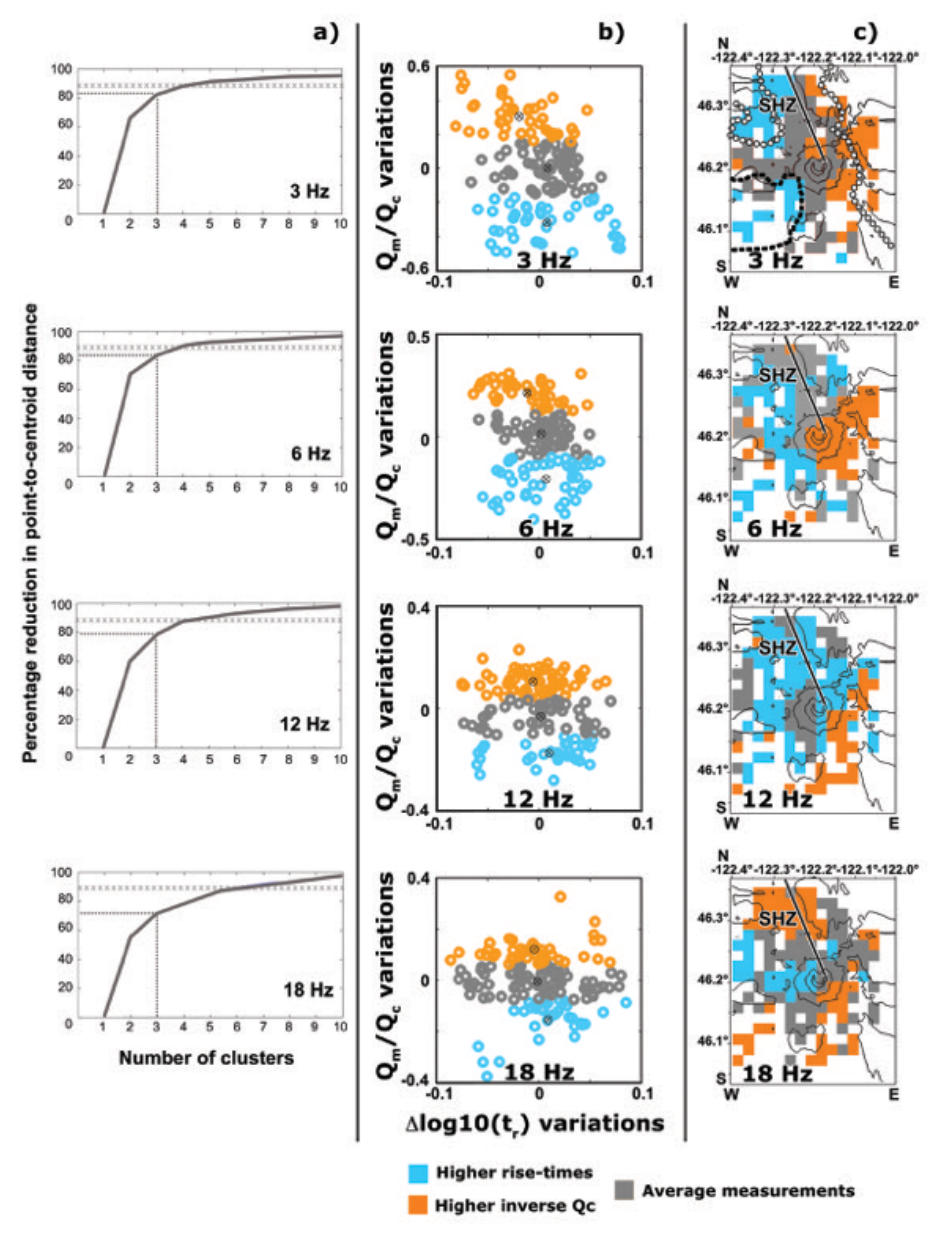

Figure 8: Results of the cluster analysis. 3-means cluster analysis applied to the 2D spatially-dependent measurements of Fig. 5a,b. a) Percent reduction in point-to-centroid distance with respect to the number of clusters for the 4 frequency bands. The x-crossed line shows the $90 \%$ reduction. The dotted line shows the percent reduction for three clusters. The centroid of each cluster (black crossed circles) and the result of the cluster analysis are shown in the parameter space (b) and mark different regions on the contour map (c). On the $3 \mathrm{~Hz}$ plots we impose the contour of high magnetic anomalies (white dotted curves) and the area of highest surface, magnetic, and depth-dependent velocity heterogeneity (black dashed line) as reported by Lees and Crosson [24]. 